\title{
Journal of Cardiovascular Magnetic

\section{Quantitative assessment of left ventricular function and volumes by cardiac MRI after application of G-CSF in postmyocarditic mice}

\author{
Alexandra Keithahn*1, Stefan Brunner ${ }^{2}$, Marcus Makowski1, \\ Rebekka Fischer ${ }^{2}$, Hans Theiss ${ }^{2}$, Wolfgang Franz ${ }^{2}$, Markus Schwaiger ${ }^{1}$ and \\ Rene Botnar ${ }^{1}$
}

Address: ${ }^{1}$ Technische Universität München, Munich, Germany and ${ }^{2}$ Ludwig Maximilians Universität München, Munich, Germany

* Corresponding author

from I th Annual SCMR Scientific Sessions

Los Angeles, CA, USA. I-3 February 2008

Published: 22 October 2008

Journal of Cardiovascular Magnetic Resonance 2008, I0(SuppI I):A372 doi:10.1 186/1532-429X-10-SI-A372

This abstract is available from: http://jcmr-online.com/content//0/SI/A372

(C) 2008 Keithahn et al; licensee BioMed Central Ltd.

\section{Background}

This pilot study was performed to evaluate left ventricular function and volumes in a murine myocarditis model before and after G-CSF-therapy on a clinical MRI scanner.

\section{Introduction}

With the advent of virally induced myocarditis models in mice, in-vivo investigation of structural, functional, and molecular changes of cardiomyopathy leading to heart failure has become feasible. Such models offer great potential for quantitative and serial assessment of ejection fraction (EF), volumes and regional wall motion. Furthermore, histological correlates can be obtained for microscopic validation.

Optimized clinical MR protocols for murine imaging on a $1.5 \mathrm{~T}$ clinical scanner were used for quantification of left ventricular function and hemodynamics before and after the application of G-CSF in a postmyocarditic mouse model.

\section{Methods}

For reliable ECG synchronization and high resolution imaging, a dedicated small animal ECG device, 1025-MR, (SA Instruments Inc., Stony Brook, NY) and a microscopy coil (Philips Medical Systems, Best, NL) were used. All imaging was performed on a $1.5 \mathrm{~T}$ Philips Achieva MR scanner using a clinical gradient system $(30 \mathrm{mT} / \mathrm{m}, 150$ $\mathrm{mT} / \mathrm{m} / \mathrm{ms}$ ). Animals were anesthetized with fentanyl $(0,05 \mathrm{mg} / \mathrm{kg})$, medetomidin $(0,5 \mathrm{mg} / \mathrm{kg})$ and midazolam $(5 \mathrm{mg} / \mathrm{kg})$ and imaged in prone position with the thorax positioned on top of the microscopy single loop surface coil $(\mathrm{D}=2.3 \mathrm{~mm})$. Cine MRI was performed with prospective ECG triggering using a spoiled gradient echo technique. Imaging parameters included TR/TE $=18 \mathrm{~ms} / 6.5$ $\mathrm{ms}$, flip angle $=30^{\circ}$, averages $=1, \mathrm{FOV}=35 \mathrm{~mm}$, matrix $=$ 128 resulting in a spatial resolution of $0.22 \times 0.22 \times 1 \mathrm{~mm}$ at a temporal resolution of $18 \mathrm{~ms}$.

Two months after coxsackievirus B3 (CVB3)-induced myocarditis 23 SWR mice where examined by cardiac MRI as discribed above. After this baseline examination, a follow up scan was performed 14 days later. Afterwords mice were divided into the following groups: 1 ) subcutaneous administration of saline daily for ten days $(\mathrm{N}=11) ; 2)$ administration of G-CSF $(100 \mu \mathrm{g} / \mathrm{kg} /$ day Amgen Biologicals) daily for 10 consecutive days $(\mathrm{N}=12)$. For comparison additional 5 SWR mice were investigated.

Image analysis was performed using an in-house developed IDL 6.1. based software package for evaluation of ejection fraction, lv-mass and hemodynamic parameters in short axis view. 


\section{Results}

At baseline 23 mice were successfully investigated be cine MRI. All mice showed a visually globally decreased wall motion. At baseline $(\mathrm{N}=23)$ ejection fraction based on short axis images was calculated 41,03\% $(+/-4,65$ mean $\mathrm{SD})$. In control mice $(\mathrm{N}=5) \mathrm{EF}$ was $48,45 \%(+/-5,42$ mean SD). Follow up scan could successfully be performed in $91 \%$ of the animals. Failure of scanning was related to an insufficient ECG signal due to arrhythmia in two animals (one in the treated group, one in the non treated group). The untreated group showed an ejection fraction of $39,84 \%(+/-5,29$ mean SD) and treated animals showed an ejection fraction of 43,81 (+/- 1,63 mean $\mathrm{SD})$ at follow up. There had been a significant increase in ejection fraction in the G-CSF treated group compared to control. Other hemodynamic parameters and lv-mass showed no significant differences using t-test for independent samples.

\section{Conclusion}

In this pilot study lv-function and mass was evaluated in 23 mice two months after induction of myocarditis by CBV-3 infection. At baseline all animals showed a modest decreased ejection-fraction and volumes compared to control. For G-CSF treated mice an increased ejection fraction could be demonstrated in comparison to control at follow up. It has to be mentioned that the number of control is low and further examinations have to be done to confirm the demonstrated positive effect on left ventricular function after the induction of myocarditis and application of G-CSF in a murin model.

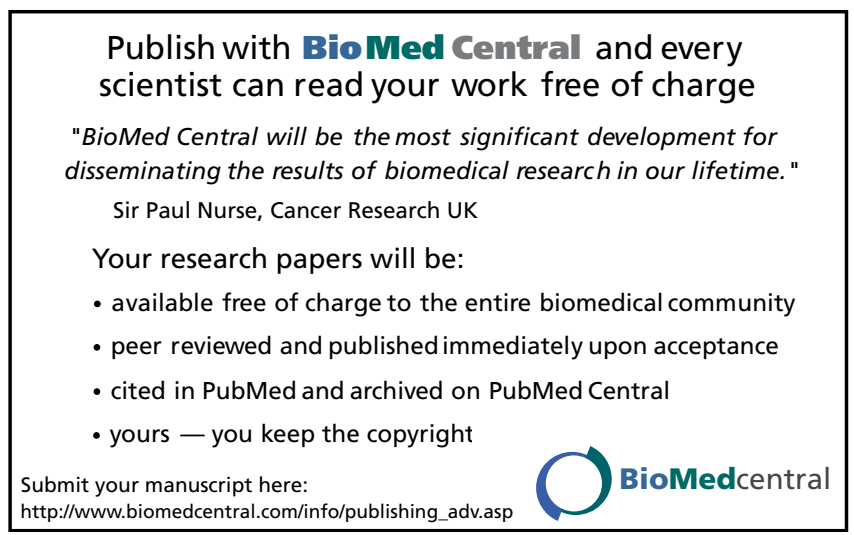

